Brief Report

\title{
Larval Exposure to the Bacterial Insecticide Bti Enhances Dengue Virus Susceptibility of Adult Aedes aegypti Mosquitoes
}

\author{
Isabelle Moltini-Conclois ${ }^{1,2,+}$, Renaud Stalinski ${ }^{3,+}$, Guillaume Tetreau ${ }^{4}\left(\mathbb{D}\right.$, Laurence Després ${ }^{3}$ \\ and Louis Lambrechts $1,2, * \mathbb{D}$ \\ 1 Insect-Virus Interactions Group, Department of Genomes and Genetics, Institut Pasteur, 75015 Paris, France; \\ isabelle.conclois@gmail.com \\ 2 Centre National de la Recherche Scientifique, Génomique Evolutive, Modélisation et Santé, UMR 2000, \\ 75015 Paris, France \\ 3 Laboratoire d'Ecologie Alpine, Université Grenoble Alpes, Centre National de la Recherche Scientifique, \\ UMR 5553, 38058 Grenoble, France; renaud.stalinski@gmail.com (R.S.); \\ laurence.despres@univ-grenoble-alpes.fr (L.D.) \\ 4 Institut de Biologie Structurale, Université Grenoble Alpes, Commissariat à l'Energie Atomique, \\ Centre National de la Recherche Scientifique, UMR 5075, 38058 Grenoble, France; \\ guillaume.tetreau@gmail.com \\ * Correspondence: louis.lambrechts@pasteur.fr \\ + These authors contributed equally to this work.
}

Received: 12 November 2018; Accepted: 12 December 2018; Published: 14 December 2018

\begin{abstract}
Understanding the interactions between pathogens sharing the same host can be complicated for holometabolous animals when larval and adult stages are exposed to distinct pathogens. In medically important insect vectors, the effect of pathogen exposure at the larval stage may influence susceptibility to human pathogens at the adult stage. We addressed this hypothesis in the mosquito Aedes aegypti, a major vector of arthropod-borne viruses (arboviruses), such as the dengue virus (DENV) and the chikungunya virus (CHIKV). We experimentally assessed the consequences of sub-lethal exposure to the bacterial pathogen Bacillus thuringiensis subsp. israelensis (Bti), during larval development, on arbovirus susceptibility at the adult stage in three Ae. aegypti strains that differ in their genetic resistance to Bti. We found that larval exposure to Bti significantly increased DENV susceptibility, but not CHIKV susceptibility, in the Bti-resistant strains. However, there was no major difference in the baseline arbovirus susceptibility between the Bti-resistant strains and their $B t i$-susceptible parental strain. Although the generality of our results remains to be tested with additional arbovirus strains, this study supports the idea that the outcome of an infection by a pathogen depends on other pathogens sharing the same host even when they do not affect the same life stage of the host. Our findings may also have implications for Bti as a mosquito biocontrol agent, indicating that the sub-optimal Bti efficacy may have counter-productive effects by increasing vector competence, at least for some combinations of arbovirus and mosquito strains.
\end{abstract}

Keywords: Bacillus thuringiensis subsp. israelensis; carry-over effect; arbovirus; vector competence; multiple infection

\section{Introduction}

Although host-pathogen interactions are often studied as a one-to-one relationship, most hosts are simultaneously or sequentially infected with several pathogens [1]. Because different infections are not necessarily independent from each other, infection probability and dynamics should be evaluated 
in the context of pathogen communities [2,3]. Pathogens sharing the same host may interact directly through competition for resources, or indirectly via the host immune response [4,5].

Understanding interactions between pathogens infecting the same host can be complicated for holometabolous animals (i.e., with complete metamorphosis) when larval and adult stages live in separate habitats and are exposed to distinct pathogens. Larval traits can have multiple, complex fitness consequences that persist across the metamorphic boundary [6]. In mosquito vectors of human pathogens, for instance, conditions experienced at the larval stage can influence adult traits underlying vectorial capacity, such as pathogen susceptibility and lifespan [7-9].

Here, we investigated whether such a 'carry-over' effect of exposure to a larval pathogen influences susceptibility to another pathogen at the adult stage. We used the mosquito Aedes aegypti, a major vector of several medically significant arthropod-borne viruses (arboviruses). Mosquitoes are holometabolous insects whose larvae develop in water prior to their metamorphosis into flying adults. We examined the consequences of a sub-lethal exposure to the bacterial pathogen Bacillus thuringiensis subsp. israelensis (Bti) during larval development on susceptibility to the dengue virus (DENV) and the chikungunya virus (CHIKV) at the adult stage.

$B t i$ is a natural mosquito pathogen known for its environmental safety and used as a biological control agent [10]. Bti is currently used worldwide as an alternative to chemical insecticides, particularly in Europe where it is the main insecticide used against mosquito larvae outdoors. During sporulation, Bti produces four main toxins: Cry4Aa, Cry4Ba, Cry11Aa and Cyt1Aa. Upon ingestion by larvae, the toxins form pores in the midgut epithelium, allowing invasion by $\mathrm{Bti}$ and gut bacteria and resulting in larval death [11].

Aedes species and populations vary in their natural susceptibility to Bti [12,13]. High levels of resistance to each individual Cry toxin of Bti can evolve in only a few generations of selection in the laboratory, but they only confer a moderate overall resistance to Bti [14,15]. Genetic resistance to Bti is associated with constitutive changes in the expression of immune genes $[16,17]$. Mosquito life-history traits (survival, development time, fecundity) were found to respond to Bti selection, suggesting that Bti-resistance alleles may have pleiotropic effects throughout the mosquito life cycle [18].

In the present study, we hypothesized that immunological or physiological changes in mosquitoes exposed to Bti toxins at immature stages might affect virus-mosquito interactions and thus arbovirus susceptibility at the adult stage. To account for the genetic differences in Bti susceptibility, we compared the consequences of sub-lethal Bti exposure in three different Ae. aegypti strains with different levels of $B t i$ resistance. The Bora-Bora strain is naturally susceptible to $B t i$, and the LR4A and LR3Bti strains were obtained by artificial selection of the Bora-Bora strain for Cry4Aa and Bti resistance, respectively [15].

\section{Materials and Methods}

\subsection{Mosquitoes}

The three Ae. aegypti strains used in this study (Bora-Bora, LR4A, LR3Bti) have been described elsewhere [15]. Briefly, mosquitoes of the Bti-susceptible Bora-Bora strain were selected during 33 generations for resistance to one of the three Cry toxins (Cry4Aa, Cry4Ba, Cry11Aa). The LR4A strain is 1018-fold more resistant to Cry4Aa than the parental Bora-Bora strain, but displays little cross-resistance to the other Cry toxins ( $<4$-fold cross-resistance to Cry4Ba and Cry11Aa). The composite LR3Bti strain was initially created by mixing $30 \%$ of each of the three resistant strains (i.e., selected for resistance to individual $B t i$ toxins) and $10 \%$ of the Bora-Bora strain, followed by 14 generations of selection for $B t i$ resistance [19]. The LR3Bti strain is 5- to 14-fold more resistant to individual Bti toxins and 1.4-fold more resistant to $B t i$ than the Bora-Bora strain. The mosquitoes were reared under standard insectary conditions $\left(27^{\circ} \mathrm{C}, 70 \%\right.$ relative humidity and a $12 \mathrm{~h}$ light: $12 \mathrm{~h}$ dark cycle) as previously described [20]. 


\subsection{Bti Exposure}

Eggs from each of the three strains were hatched under reduced air pressure for $1 \mathrm{~h}$. Because the LR4A and LR3Bti strains develop slower than the Bora-Bora strain, their eggs were hatched $24 \mathrm{~h}$ earlier to synchronize adult emergence. After hatching, 200 first-instar larvae from each strain were sorted into $24 \times 34 \times 9 \mathrm{~cm}$ plastic trays filled with $1.5 \mathrm{~L}$ of dechlorinated tap water. The larvae were fed on a standard diet of Tetramin fish food (Tetra) for 4-5 days. Groups of 100 third-instar larvae from each strain were placed into Coplin jars containing $30 \mathrm{mg}$ of fish food (Tetra). They were exposed (treatment) or non-exposed (control) to a dose of Bti (toxins and spores) adjusted to reach the $50 \%$ lethal concentration ( $\mathrm{LC}_{50}$ ) of each strain $(151.9 \mu \mathrm{g} / \mathrm{mL}$ for the Bora-Bora strain and $216.2 \mu \mathrm{g} / \mathrm{mL}$ for the LR4A and LR3Bti strains). After $24 \mathrm{~h}$ of exposure to Bti, the surviving fourth-instar larvae were returned to their rearing trays. The number of larval rearing trays was 6, 9 and 12 for the Bora-Bora, LR4A and LR3Bti strains, respectively. The number of Coplin jars for Bti exposure was 12, 18 and 24 for the Bora-Bora, LR4A and LR3Bti strains, respectively. The pupae were transferred to cages and the emerging adults were maintained under standard insectary conditions with permanent access to $10 \%$ sucrose solution.

\subsection{Arbovirus Exposure}

Following Bti exposure at the larval stage, 5- to 8-day-old adult females were exposed to the dengue virus (DENV; type 1 strain KDH0030A [21]) or the chikungunya virus (CHIKV; strain 06-21 [22]) in an artificial infectious blood meal as previously described [20]. For each treatment and arbovirus, the adults were exposed to the infectious blood meal in 1,2-3 and 2 boxes of 60 females for the Bora-Bora, LR4A and LR3Bti strains, respectively. The blood feeding rate was $51.7 \%$ on average and ranged from $25.8 \%$ to $68.3 \%$ between the boxes. The rabbit blood draws performed in the context of this study were approved by the Institutional Animal Care and Use Committee at Institut Pasteur, under protocol number 2015-0032. The virus stocks were prepared in C6/36 (Aedes albopictus) cells and titrated by fluorescent focus-forming assay (FFA) as previously described [20]. The final blood meal titer was $4.75 \times 10^{5}$ focus-forming units (FFU) $/ \mathrm{mL}$ for DENV and $5.38 \times 10^{5} \mathrm{FFU} / \mathrm{mL}$ for CHIKV.

\subsection{Arbovirus Susceptibility}

Susceptibility to DENV and CHIKV was measured by (i) the proportion of females that became infected (infection rate) and (ii) the proportion of infected mosquitoes in which the virus disseminated to the head tissues (dissemination rate). After 7 days (CHIKV) and 10 days (DENV) of incubation, mosquitoes were harvested and dissected. The heads were separated from their bodies with a clean scalpel that was decontaminated between each individual with Hexanios $G+R$ $0.5 \%$ (Anios). The two time points were chosen to reflect the difference in the viral dissemination kinetics between CHIKV and DENV [23]. The infection rates were measured by the detection of viral RNA in the mosquito bodies. The dissemination rates were measured by detection of the infectious virus in the head tissues of body-positive mosquitoes. The detection of DENV RNA in the body samples was performed by reverse transcription (RT) quantitative PCR as previously described [20]. The detection of CHIKV RNA in the body samples was performed by RT-PCR as follows. The total RNA was reverse-transcribed to complementary DNA (cDNA) with random hexamers. The cDNA was amplified by PCR (forward primer: 5'-AAGCTYCGCGTCCTTTACCAAG-3'; reverse primer: $5^{\prime}$-CCAAATTGTCCYGGTCTTCCT-3'). The amplicons were visualized by electrophoresis on $2 \%$ agarose gels. For both DENV and CHIKV, the heads were homogenized individually and processed for infectious virus detection using a qualitative version of the FFA [20] that only tests the undiluted sample and therefore does not allow end-point titration. 


\subsection{Bti Persistence}

To measure Bti persistence at the adult stage, 4-day-old adult males and females from the LR4A and LR3Bti strains that survived $24 \mathrm{~h}$ of Bti exposure $\left(\mathrm{LC}_{50}\right)$ at the third-instar larval stage were homogenized in $200 \mu \mathrm{L}$ PBS. In order to determine whether Bti could persist as spores and/or as vegetative cells throughout mosquito development, the homogenates were divided into two parts. One half was heated for $60 \mathrm{~min}$ at $80{ }^{\circ} \mathrm{C}$, in order to kill most bacteria except the Bti spores that survive this treatment [24]. Both the heated and non-heated homogenates were plated separately on nutrient agar. After $24 \mathrm{~h}$ at $30^{\circ} \mathrm{C}$, the bacterial colonies were identified and counted.

\subsection{Statistical Analyses}

The proportion of infected mosquitoes and the proportion of infected mosquitoes with a disseminated infection were analyzed as binary response variables (Table S1) using logistic regression. The treatment (Bti vs. unexposed) and strain (LR3Bti, LR4A, Bora-Bora) were included as covariates in a full-factorial model. The pairwise differences were assessed by likelihood-ratio $\chi^{2}$ tests, followed by Bonferroni correction for multiple testing. All analyses were performed in JMP v10 (SAS Institute Inc., Cary, NC, USA).

\section{Results}

Following sub-lethal Bti exposure at the larval stage, a total of 184 (57-64 per strain) Ae. aegypti females were orally challenged with DENV. Overall, $65.8 \%$ of the mosquitoes became infected with DENV and $49.6 \%$ of the infected mosquitoes developed a disseminated infection. DENV infection was influenced by a strong interaction between the strain and the treatment (Table 1). In pairwise comparisons, DENV infection was significantly higher in the Bti-exposed mosquitoes of the LR3Bti $(p=0.027)$ and LR4A $(p=0.0006)$ strains relative to the unexposed controls, whereas there was no effect of Bti exposure in the Bora-Bora strain (Figure 1). The DENV dissemination rate was also influenced by a strong interaction between the strain and the treatment (Table 1). In pairwise comparisons, DENV dissemination was significantly higher in the Bti-exposed mosquitoes of the LR3Bti strain ( $p=0.0303$ ), but there was no effect of Bti exposure in the LR4A and Bora-Bora strains (Figure 1).

Table 1. Test statistics of arbovirus susceptibility phenotypes.

\begin{tabular}{cccccc}
\hline & & \multicolumn{2}{c}{ Infection } & \multicolumn{2}{c}{ Dissemination } \\
\cline { 3 - 6 } & df & LR $\chi^{2}$ & $p$ Value & LR $\chi^{2}$ & $p$ Value \\
\hline DENV & & & & & \\
\hline Strain & 2 & 13.5 & 0.0012 & 0.57 & 0.7527 \\
Treatment & 1 & 12.5 & 0.0004 & 0.03 & 0.8562 \\
Strain $\times$ & 2 & 11.8 & 0.0027 & 9.25 & 0.0098 \\
Treatment & 2 & & & & \\
\hline CHIKV & & & & \\
\hline Strain & 2 & 6.82 & 0.0331 & 0.69 & 0.7068 \\
Treatment & 1 & 0.60 & 0.4401 & 2.17 & 0.1404 \\
Strain $\times$ & 2 & 5.58 & 0.0613 & 4.26 & 0.1187 \\
Treatment & & & &
\end{tabular}




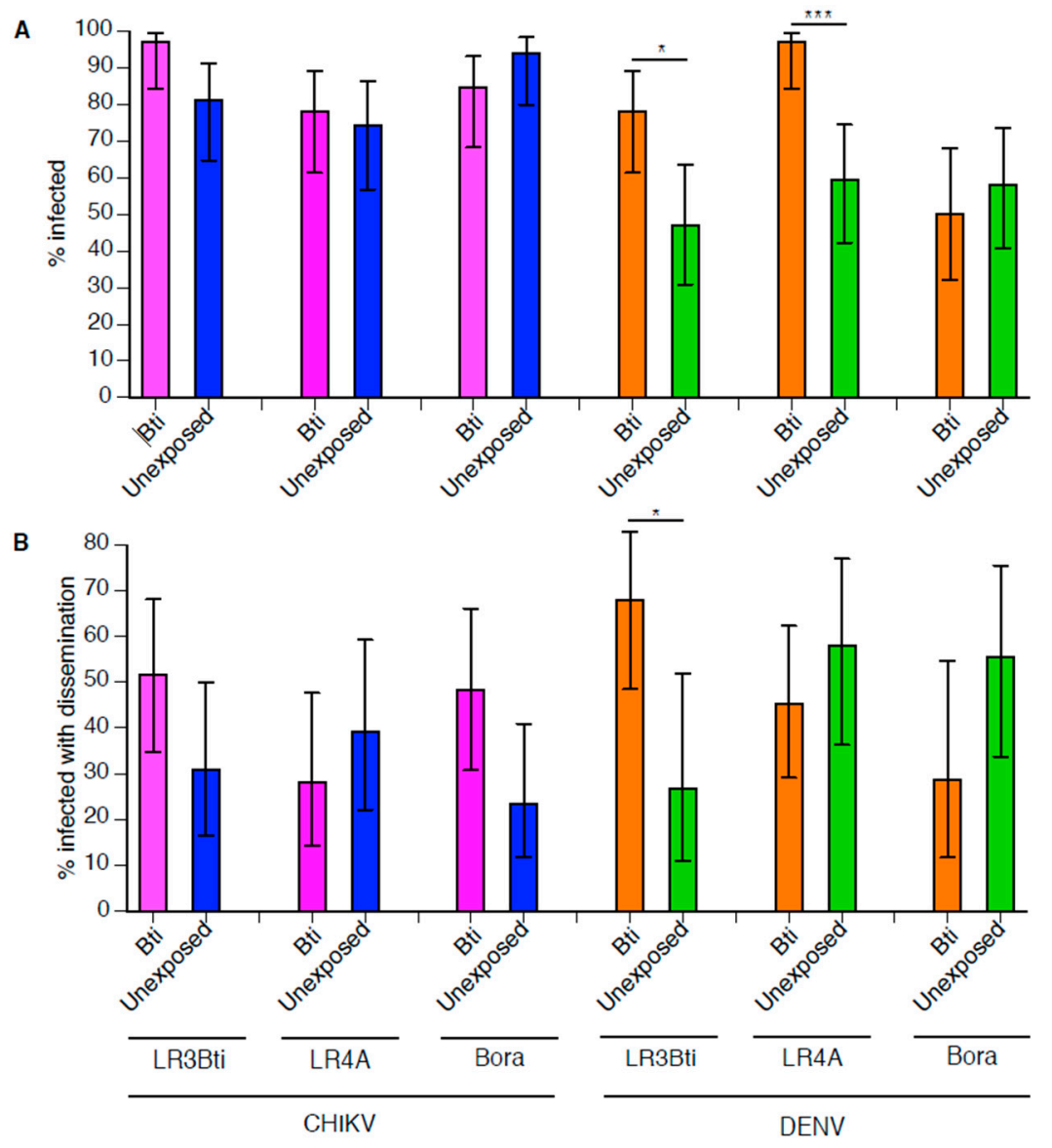

Figure 1. Arbovirus susceptibility following sub-lethal Bacillus thuringiensis subsp. israelensis (Bti) exposure during larval development. For each arbovirus, the chikungunya virus (CHIKV) and the dengue virus (DENV), the percentage of infected mosquitoes (A) and the percentage of infected mosquitoes with a disseminated infection (B) are shown as a function of strain and treatment. Error bars represent $95 \%$ confidence intervals of the percentages. Stars above the bars indicate statistical significance of pairwise differences after Bonferroni correction for multiple testing $\left({ }^{*} p<0.05 ;{ }^{* *} p<0.01\right.$; *** $p<0.001)$. Test statistics for the full model are provided in Table 1.

Following sub-lethal Bti exposure at the larval stage, a total of 191 (63-64 per strain) Ae. aegypti females were orally challenged with CHIKV. Overall, $84.8 \%$ of the mosquitoes became infected with CHIKV and $37.0 \%$ of the infected mosquitoes developed a disseminated infection. The CHIKV infection rate was only marginally significantly influenced by the strain (Table 1), due to a slightly lower infection rate of LR4A mosquitoes, irrespective of the Bti treatment. There was no significant effect of the $B t i$ treatment or interaction between the strain and the treatment on the CHIKV infection rates (Table 1; Figure 1). The CHIKV dissemination rate was not significantly influenced by the strain, the treatment, or their interaction (Table 1; Figure 1).

A total of 18 adults from the LR4A $(n=3)$ and LR3Bti $(n=15)$ strains were tested for Bti persistence at the adult stage. None of them were found positive for $B t i$.

\section{Discussion}

We tested whether sub-lethal pathogen exposure at the larval stage would influence pathogen susceptibility at the adult stage in a holometabolous insect. We found evidence supporting this hypothesis in the mosquito vector Ae. aegypti, although it depended on the mosquito strain and the infecting pathogen. Exposure to the bacterial pathogen Bti at the larval stage subsequently enhanced adult susceptibility to DENV, but not CHIKV, and only in the Bti-resistant strains, not in the 
Bti-susceptible strain. However, there was no evidence for an association between the level of genetic resistance to Bti and intrinsic DENV or CHIKV susceptibility. Note that the lack of significant effect for CHIKV in our experiment could be due to the higher overall infection rate across the strains and treatments $(84.8 \%)$ than for DENV $(65.8 \%)$. This may have reduced our power to detect an increase in the CHIKV infection rates between the strains and treatments.

Although these results will need to be followed up with additional experiments using different arbovirus strains [25], they support the idea that the outcome of host infection by a given pathogen depends on other pathogens sharing the same host $[2,4]$. They go one step further by showing that this can be the case even when pathogens do not affect the same life stage. Although the underlying mechanism is unknown, the observed carry-over effect is unlikely to result from direct competition because Bti did not persist at the adult stage when the mosquitoes were orally challenged with the DENV. We speculate that it more likely results from indirect effects, such as tissue damage, immune impairment, or reserve depletion.

Our results differ from a previous study reporting that following Bti exposure at the larval stage, Ae. aegypti females had similar rates of DENV infection and dissemination than unexposed controls [7]. This discrepancy could be due to several factors. Firstly, we used laboratory strains of Ae. aegypti, whereas the earlier study used a field-derived population. We observed that the response to Bti exposure differed among Ae. aegypti strains, indicating that the effect of Bti varies across mosquito genotypes. Secondly, we exposed third-instar larvae to their Bti $\mathrm{LC}_{50}$, whereas in the earlier study first-instar larvae were exposed to concentrations of Bti that were 4- to 24-fold lower than their LC $_{50}$. Thirdly, the effect could be dependent on the DENV strain, which was not the same between the studies.

Our protocol eliminated $50 \%$ of individuals at the larval stage and therefore the carry-over effect that we observed could be confounded with natural selection. In other words, non-random elimination of $50 \%$ of the individuals could contribute to explaining differences in DENV susceptibility that are not a direct consequence of sub-lethal Bti exposure, but rather are due to the selection of genetically more-susceptible individuals. However, because the Bti-resistant strains were selected for at least 14 generations prior to this experiment [15], it is unlikely that one additional generation of selection would have substantially changed the genetic composition of these strains.

Although the Bti-resistant strains exhibit both different gene expression profiles and specific polymorphisms relative to their Bti-susceptible parental strain [26], we did not observe major differences in the baseline arbovirus susceptibility (in the absence of Bti exposure) between the LR4A, LR3Bti and Bora-Bora strains. However, the Bti-resistant strains displayed higher DENV susceptibility at the adult stage after Bti exposure at the larval stage, suggesting that Bti-selected genetic changes may have contributed to the carry-over effect. In addition to putative Cry-toxins receptors, many genes involved in the cuticle/chitin metabolism and in gut enzymatic functions (e.g., detoxification, trans-membrane transport, proteolytic activities) were constitutively differently expressed in the $B t i$-resistant strains relative to the Bora-Bora strain $[17,26]$. Interestingly, two specific antimicrobial peptides were over-expressed in the Bora-Bora strain upon Bti exposure [15], however this response has not been investigated in the Bti-resistant strains. Another important consequence of Ae. aegypti exposure to Bti is a dramatic change in the composition of the larval gut bacterial microbiome [27], which could have mediated the carry-over effect on DENV susceptibility [8].

Finally, our findings have potential implications for Bti as a mosquito biocontrol agent. Exposure to a sub-lethal dose of Bti increased DENV susceptibility in the surviving adults of the Bti-resistant strains, potentially canceling out the larvicidal effect by increasing permissiveness to arbovirus infection in the surviving individuals. Although other parameters, such as adult mosquito density and survival, have to be taken into account to quantitatively estimate the net effect of Bti on vectorial capacity $[7,8]$, our results indicate that the sub-optimal Bti efficacy may have counter-productive effects by increasing vector competence. 


\section{Conclusions}

Although the generality of our results remains to be tested with additional arbovirus strains, this study supports the idea that the outcome of an infection by a pathogen depends on other pathogens sharing the same host even when they do not affect the same life stage of the host. Our results also have implications for $B t i$ as a mosquito biocontrol agent, because they indicate that Bti may have counter-productive effects on vectorial capacity when its efficacy is sub-optimal.

Supplementary Materials: The following are available online at http:/ /www.mdpi.com/2075-4450/9/4/193/s1: Table S1. Raw dataset. For each strain, treatment and arbovirus, the susceptibility phenotypes are indicated $(0=$ uninfected; 1 = infected $)$.

Author Contributions: L.L. and L.D. conceived the study. All authors designed the experiments. I.M.-C. and R.S. performed the experiments. L.L. analyzed the data and wrote the manuscript. L.D. and G.T. helped draft the manuscript. All authors gave final approval for publication.

Funding: This work was supported by Agence Nationale de la Recherche (grant ANR-16-CE35-0004-01 to L.L.), the French Government's Investissement d'Avenir program Laboratoire d'Excellence Integrative Biology of Emerging Infectious Diseases (grant ANR-10-LABX-62-IBEID to L.L.), the Région Rhône-Alpes (CIBLE program RA0000R464 to L.D.) and the City of Paris Emergence(s) program (to L.L.).

Acknowledgments: We thank Catherine Lallemand and Frédéric Laporte for assistance with mosquito rearing, Anna-Bella Failloux for sharing the CHIKV isolate, and three anonymous reviewers for constructive comments on an earlier version of the manuscript.

Conflicts of Interest: The authors declare no conflict of interest. The sponsors had no role in the design, execution, interpretation, or writing of the study.

\section{References}

1. Cox, F.E.G. Concomitant infections, parasites and immune responses. Parasitology 2001, 122 (Suppl. S1), S23-S38. [CrossRef] [PubMed]

2. Telfer, S.; Lambin, X.; Birtles, R.; Beldomenico, P.; Burthe, S.; Paterson, S.; Begon, M. Species interactions in a parasite community drive infection risk in a wildlife population. Science 2010, 330, 243-246. [CrossRef]

3. Rynkiewicz, E.C.; Pedersen, A.B.; Fenton, A. An ecosystem approach to understanding and managing within-host parasite community dynamics. Trends Parasitol. 2015, 31, 212-221. [CrossRef] [PubMed]

4. Graham, A.L. Ecological rules governing helminth-microparasite coinfection. Proc. Natl. Acad. Sci. USA 2008, 105, 566-570. [CrossRef] [PubMed]

5. Pedersen, A.B.; Fenton, A. Emphasizing the ecology in parasite community ecology. Trends Ecol. Evol. 2007, 22, 133-139. [CrossRef] [PubMed]

6. Crean, A.J.; Monro, K.; Marshall, D.J. Fitness consequences of larval traits persist across the metamorphic boundary. Evolution 2011, 65, 3079-3089. [CrossRef]

7. Alto, B.W.; Lord, C.C. Transstadial effects of Bti on traits of Aedes aegypti and infection with dengue virus. PLoS Negl. Trop. Dis. 2016, 10, e0004370. [CrossRef]

8. Dickson, L.B.; Jiolle, D.; Minard, G.; Moltini-Conclois, I.; Volant, S.; Ghozlane, A.; Bouchier, C.; Ayala, D.; Paupy, C.; Moro, C.V.; et al. Carryover effects of larval exposure to different environmental bacteria drive adult trait variation in a mosquito vector. Sci. Adv. 2017, 3, e1700585. [CrossRef]

9. Roux, O.; Vantaux, A.; Roche, B.; Yameogo, K.B.; Dabire, K.R.; Diabate, A.; Simard, F.; Lefevre, T. Evidence for carry-over effects of predator exposure on pathogen transmission potential. Proc. Biol. Sci. 2015, 282, 20152430. [CrossRef]

10. Lacey, L.A. Bacillus thuringiensis serovariety israelensis and Bacillus sphaericus for mosquito control. J. Am. Mosq. Control. Assoc. 2007, 23, 133-163. [CrossRef]

11. Vachon, V.; Laprade, R.; Schwartz, J.L. Current models of the mode of action of Bacillus thuringiensis insecticidal crystal proteins: A critical review. J. Invertebr. Pathol. 2012, 111, 1-12. [CrossRef] [PubMed]

12. Paris, M.; Marcombe, S.; Coissac, E.; Corbel, V.; David, J.P.; Despres, L. Investigating the genetics of Bti resistance using mRNA tag sequencing: Application on laboratory strains and natural populations of the dengue vector Aedes aegypti. Evol. Appl. 2013, 6, 1012-1027. [PubMed] 
13. Tetreau, G.; Stalinski, R.; David, J.P.; Despres, L. Monitoring resistance to Bacillus thuringiensis subsp. israelensis in the field by performing bioassays with each Cry toxin separately. Mem. Inst. Oswaldo Cruz 2013, 108, 894-900. [CrossRef] [PubMed]

14. Paris, M.; Tetreau, G.; Laurent, F.; Lelu, M.; Despres, L.; David, J.P. Persistence of Bacillus thuringiensis israelensis (Bti) in the environment induces resistance to multiple Bti toxins in mosquitoes. Pest Manag. Sci. 2011, 67, 122-128. [CrossRef] [PubMed]

15. Stalinski, R.; Laporte, F.; Despres, L.; Tetreau, G. Alkaline phosphatases are involved in the response of Aedes aegypti larvae to intoxication with Bacillus thuringiensis subsp. israelensis Cry toxins. Environ. Microbiol. 2016, 18, 1022-1036. [PubMed]

16. Despres, L.; Stalinski, R.; Faucon, F.; Navratil, V.; Viari, A.; Paris, M.; Tetreau, G.; Poupardin, R.; Riaz, M.A.; Bonin, A.; et al. Chemical and biological insecticides select distinct gene expression patterns in Aedes aegypti mosquito. Biol. Lett. 2014, 10, 20140716. [CrossRef] [PubMed]

17. Despres, L.; Stalinski, R.; Tetreau, G.; Paris, M.; Bonin, A.; Navratil, V.; Reynaud, S.; David, J.P. Gene expression patterns and sequence polymorphisms associated with mosquito resistance to Bacillus thuringiensis israelensis toxins. BMC Genomics 2014, 15, 926. [CrossRef] [PubMed]

18. Paris, M.; David, J.P.; Despres, L. Fitness costs of resistance to Bti toxins in the dengue vector Aedes aegypti. Ecotoxicology 2011, 20, 1184-1194. [CrossRef]

19. Stalinski, R.; Tetreau, G.; Gaude, T.; Després, L. Pre-selecting resistance against individual Bti Cry toxins facilitates the development of resistance to the Bti toxins cocktail. J. Invertebr. Pathol. 2014, 119, 50-53. [CrossRef]

20. Fontaine, A.; Jiolle, D.; Moltini-Conclois, I.; Lequime, S.; Lambrechts, L. Excretion of dengue virus RNA by Aedes aegypti allows non-destructive monitoring of viral dissemination in individual mosquitoes. Sci. Rep. 2016, 6, 24885. [CrossRef]

21. Fansiri, T.; Fontaine, A.; Diancourt, L.; Caro, V.; Thaisomboonsuk, B.; Richardson, J.H.; Jarman, R.G.; Ponlawat, A.; Lambrechts, L. Genetic mapping of specific interactions between Aedes aegypti mosquitoes and dengue viruses. PLoS Genet. 2013, 9, e1003621. [CrossRef] [PubMed]

22. Schuffenecker, I.; Iteman, I.; Michault, A.; Murri, S.; Frangeul, L.; Vaney, M.C.; Lavenir, R.; Pardigon, N.; Reynes, J.M.; Pettinelli, F.; et al. Genome microevolution of chikungunya viruses causing the Indian Ocean outbreak. PLoS Med. 2006, 3, e263. [CrossRef]

23. Ruckert, C.; Weger-Lucarelli, J.; Garcia-Luna, S.M.; Young, M.C.; Byas, A.D.; Murrieta, R.A.; Fauver, J.R.; Ebel, G.D. Impact of simultaneous exposure to arboviruses on infection and transmission by Aedes aegypti mosquitoes. Nat. Commun. 2017, 8, 15412. [CrossRef] [PubMed]

24. Duchet, C.; Tetreau, G.; Marie, A.; Rey, D.; Besnard, G.; Perrin, Y.; Paris, M.; David, J.P.; Lagneau, C.; Despres, L. Persistence and recycling of bioinsecticidal Bacillus thuringiensis subsp. israelensis spores in contrasting environments: Evidence from field monitoring and laboratory experiments. Microb. Ecol. 2014, 67, 576-586. [CrossRef] [PubMed]

25. Lambrechts, L. Dissecting the genetic architecture of host-pathogen specificity. PLoS Pathog. 2010, 6, e1001019. [CrossRef] [PubMed]

26. Stalinski, R.; Laporte, F.; Tetreau, G.; Despres, L. Receptors are affected by selection with each Bacillus thuringiensis israelensis Cry toxin but not with the full Bti mixture in Aedes aegypti. Infect. Genet. Evol. 2016, 44, 218-227. [CrossRef]

27. Tetreau, G.; Grizard, S.; Patil, C.D.; Tran, F.H.; Tran Van, V.; Stalinski, R.; Laporte, F.; Mavingui, P.; Despres, L.; Valiente Moro, C. Bacterial microbiota of Aedes aegypti mosquito larvae is altered by intoxication with Bacillus thuringiensis israelensis. Parasit. Vectors 2018, 11, 121. [CrossRef]

(C) 2018 by the authors. Licensee MDPI, Basel, Switzerland. This article is an open access article distributed under the terms and conditions of the Creative Commons Attribution (CC BY) license (http:/ / creativecommons.org/licenses/by/4.0/). 